\title{
Thermodynamics of five-dimensional Schwarzschild black holes in the canonical ensemble
}

\author{
Rui Andre* and José P. S. Lemos \\ Centro de Astrofísica e Gravitação - CENTRA, Departamento de Física, Instituto Superior Técnico - IST, \\ Universidade de Lisboa - UL, Av. Rovisco Pais 1, 1049-001 Lisboa, Portugal
}

\begin{abstract}
We study the thermodynamics of a five-dimensional Schwarzschild black hole, also known as a fivedimensional Schwarzschild-Tangherlini black hole, in the canonical ensemble using York's formalism. Inside a cavity of fixed size $r$ and fixed temperature $T$, we find that there is a threshold at $\pi r T=1$ above which a black hole can be in thermal equilibrium with the cavity's boundary. Moreover, this thermal equilibrium can only be achieved for two specific types of black holes. One is a small, compared with the cavity size $r$, black hole of horizon radius $r_{+1}$, while the other is a large, of the order of the cavity size $r$, black hole of horizon radius $r_{+2}$. In five dimensions, the radii $r_{+1}$ and $r_{+2}$ have an exact expression. Through the path integral formalism, which directly yields the partition function of the system, one obtains the action and thus the free energy for the black hole in the canonical ensemble. The procedure leads naturally to the thermal energy and entropy of the canonical system, the latter turning out to be given by the Bekenstein-Hawking area law $S=\frac{A_{+}}{4}$, where the black hole's surface area in five dimensions is $A_{+}=2 \pi^{2} r_{+}^{3}$ and $r_{+}$stands for both $r_{+1}$ and $r_{+2}$. We also calculate the heat capacity and find that it is positive when the heat bath is placed at a radius $r$ that is equal or less than the photonic orbit, implying in this case thermodynamic stability, and instability otherwise. This means that the small black hole $r_{+1}$ is unstable and the large one $r_{+2}$ is stable. A generalized free energy is used to compare the possible thermodynamic phase transitions relative to classical hot flat space which has zero free energy, and we show that it is feasible in certain instances that classical hot flat space transits through $r_{+1}$ to settle at the stable $r_{+2}$, with the free energy of the unstable smaller black hole $r_{+1}$ acting as the potential barrier between the two states. It is also shown that, remarkably, the free energy of the larger $r_{+2}$ black hole is zero when the cavity radius is equal to the Buchdahl radius. The relation to the instabilities that arise due to perturbations in the path integral in the instanton solution is mentioned. Hot flat space is made of gravitons and it should be treated quantum mechanically rather than classically. Quantum hot flat space has negative free energy and we find the conditions for which the large black hole phase, quantum hot flat space phase, or both are the ground state of the canonical ensemble. The corresponding phase diagram is displayed in a $r \times T$ plot showing clearly the three possible phases. Using the density of states $\nu$ at a given energy $E$ we also find that the entropy of the large black hole $r_{+2}$ is $S=\frac{A_{+2}}{4}$. In addition, we make the connection between the five-dimensional thermodynamics and York's four-dimensional results.
\end{abstract}

\section{INTRODUCTION}

York substantiated the study of black hole thermodynamics by studying a four-dimensional Schwarzschild black hole in the canonical ensemble and deriving the corresponding Euclidean path integral [1. In this formalism the black hole is put in a heat bath, i.e., inside a spherical cavity that has a radius $r$ at temperature $T$.

This stemmed from the work of Hawking and collaborators 2 2-4 that used path-integral techniques to study gravitational systems containing black holes and to show that, by imposing precise boundary data on the corresponding Euclidean spacetime instanton, the black hole temperature is fixed through the correct period in imaginary time. With these techniques it was found that, in Planck units, the entropy $S$ is indeed $S=\frac{1}{4} A_{+}$, where $A_{+}$is the black hole horizon area, which comes from the contribution of the classical first-order Euclidean Einstein action of the black hole geometry to the canonical partition function. In ordinary quantum field theory the value of the classical action is absorbed into the nor-

\footnotetext{
*Electronic address: rui.andre@tecnico.ulisboa.pt

${ }^{\dagger}$ Electronic address: joselemos@tecnico.ulisboa.pt
}

malization of the functional integral and so is physically irrelevant, but in gravitational theories and, in particular, in black hole spacetimes the classical action contributes to the entropy. It was stressed in [4 that in the path-integral approach the Euler characteristic $\chi$ of the four-dimensional Euclidean black hole, which gives its topology, is the feature that gives a nonzero entropy.

One can then perturb the instanton solution of the Euclidean classical black hole through thermal quantum fluctuations and find that, while for a large cavity radius the solution is unstable [5, for a small cavity radius the solution is stable [6. This came full circle with the thermodynamic work of York 1], where in the canonical ensemble a small black hole in a given cavity is unstable, whereas a large black hole is stable. With hindsight one finds that Hawking's path-integral approach generated the correct black hole entropy, but the formalism available 22 4] only applied for the small black hole, which is unstable and thus defies a proper thermodynamic treatment.

York's method has been studied for different black holes, such as charged black holes in the grand canonical ensemble [7, 8, black holes with matter in arbitrary configurations [9], and black holes in anti-de Sitter spacetimes [10].

Another direction of study is to extend the York and 
path-integral perturbation approaches to higher dimensions [11], see also [12, 13]. Correspondingly, the pathintegral approach was also used to extend to higher dimensions the idea that the black hole entropy stems from the Euler characteristic $\chi$ of a Euclidean black hole. Indeed, in [14] it was shown that the entropy of a black hole in any dimension stems solely from the Euler characteristic of the two-dimensional plane $(r, t)$, spanned by the radial coordinate $r$ and Euclidean time $t$.

In this work we perform a full study of the thermodynamics of the five-dimensional Schwarzschild black hole, also called a Schwarzschild-Tangherlini black hole, in a canonical ensemble using York's ideas developed for the four-dimensional Schwarzschild black hole 11. The important higher-dimensional analysis done in [1] was more concerned with the stable and unstable modes of the black holes in the path-integral approach. Among several results in our thermodynamic analysis, we find the ground state of the canonical ensemble, explicitly showing for which conditions the ground state is provided by the large black hole or by quantum hot flat space. We make use of the Schwarzschild-Tangherlini metric in five dimensions [15], we mention the Buchdahl limiting radius in four [16] and higher dimensions [17, and cite the photonic orbit radius of the five-dimensional Schwarzschild-Tangherlini metric [18. For the quantum hot flat space properties in five dimensions that we need, see [19].

The paper is organized as follows. In Sec. II we construct a cavity with an interior black hole, and by fixing the cavity's size and temperature we find the possible black hole solutions that are in thermal equilibrium with the surrounding heat bath. We also compute the Euclidean Einstein-Hilbert action for a five-dimensional Schwarzschild black hole as a function of the cavity's temperature and size. In Sec. III we derive all relevant thermodynamic quantities of the black hole, write the first law of thermodynamics, and find the thermodynamic energy, pressure, and entropy of the system. In Sec. IV we compute the heat capacity in order to find which of the black hole solutions in thermal equilibrium are actually stable. In Sec. $\mathrm{V}$ we study a generalized Helmholtz free energy function, which is the thermodynamic potential of the canonical ensemble proportional to the action, and elaborate on its role in black hole nucleation from classical hot flat space, studying the corresponding instabilities. In Sec. VI we comment on the action functional to second order and its relation to thermodynamics and thermal stability. In Sec. VII we treat hot flat space in quantum terms as a bath of gravitons, and identify in which situations the black hole solution phase, the quantum hot flat space phase, or the coexistence of both phases is the ground state of the canonical ensemble, i.e., which of the phases has the lowest free energy. In Sec. VIII we find the density of states for the stable black hole solution using the partition function and manage to compute the entropy of the stable black hole. In the Appendix we comment on York's four-dimensional results and compare them with the five-dimensional results.

\section{THE CANONICAL ENSEMBLE FOR A CAVITY WITH A BLACK HOLE INSIDE: TEMPERATURE, THE EUCLIDEAN EINSTEIN ACTION, AND THE ACTION FUNCTIONAL OR PARTITION FUNCTION FOR A FIVE-DIMENSIONAL SCHWARZSCHILD BLACK HOLE}

\section{A. The cavity in five dimensions and the canonical temperature}

The temperature at infinity of a black hole is defined by Hawking's prescription for quantum fields in curved spacetimes. We use units in which the gravitational constant, the velocity of light, the Planck constant, and the Boltzmann constant are set to unity, $G=1, c=1, \hbar=1$, and $k_{B}=1$, respectively. The Schwarzschild Euclidean metric in five dimensions is given in the usual spherical coordinates by the line element [15]

$$
d s^{2}=\left(1-\frac{r_{+}^{2}}{r^{2}}\right) d t^{2}+\left(1-\frac{r_{+}^{2}}{r^{2}}\right)^{-1} d r^{2}+r^{2} d \Omega_{3}^{2}
$$

where $t$ is the Euclidean imaginary time, $r$ is the radial coordinate, the subscript 3 in $\Omega_{3}$ indicates the three angular coordinates $\theta_{1}, \theta_{2}, \theta_{3}$, with $d \Omega_{3}^{2}=d \theta_{1}^{2}+$ $\sin ^{2} \theta_{1}\left(d \theta_{2}^{2}+\sin ^{2} \theta_{2} d \theta_{3}^{2}\right)$, and $r_{+}$is the gravitational radius related in five dimensions to the spacetime's ADM mass $m$ through $r_{+}^{2}=\frac{8}{3 \pi} m$. In general, we always use $r_{+}$, not $m$. The metric describes the space outside of the event horizon $r_{+}$and is devoid of singularities, provided that the a priori conical singularity at the horizon is removed by attributing the correct period to the Euclidean time. Close to the horizon, by redefining $r=r_{+}+\varepsilon$ for small $\varepsilon$ and introducing $\rho=\sqrt{2 r_{+} \varepsilon}$, the metric in the reduced $(t, \rho)$ Euclidean space is $d s^{2}=\rho^{2}\left(\frac{d t}{r_{+}}\right)^{2}+d \rho^{2}$. This equation clearly has a Euclidean polar coordinate form, where the angular coordinate is $t / r_{+}$, and so to avoid conical singularities $t$ must have period $2 \pi r_{+}$. Thus, the Euclidean two-space has topology $R^{2}$ and the full Euclidean space has topology $R^{2} \times S^{3}$. We denote the Euclidean time period by $\beta_{\infty}$, so $\beta_{\infty}=2 \pi r_{+}$. From Hawking's prescription, this is the inverse Hawking temperature $T_{\infty}=\frac{1}{\beta_{\infty}}=\frac{1}{2 \pi r_{+}}$.

In statistical physics, the canonical ensemble is defined as the ensemble for which the temperature $T$ is kept fixed. Since our gravitational system is spherically symmetric it is natural to define the canonical ensemble as one for which the temperature $T$ is fixed at some boundary radius $r_{\mathrm{B}}$, which henceforth we abbreviate to $r$, and which should not be confounded with the usual radial coordinate $r$. This boundary at radius $r$ has topology $S^{1} \times S^{3}$, with $S^{3}$ having the usual three-surface area $2 \pi^{2} r^{3}$. From Eq. (1), $S^{1}$ has proper length $\beta$ given by $\beta=\int_{0}^{\beta_{\infty}} \sqrt{1-\frac{r_{+}^{2}}{r^{2}}} d t=\beta_{\infty} \sqrt{1-\frac{r_{+}^{2}}{r^{2}}}$, which upon using $\beta_{\infty}=2 \pi r_{+}$yields the length

$$
\beta=2 \pi r_{+} \sqrt{1-\frac{r_{+}^{2}}{r^{2}}}
$$

Imposing an isothermal boundary condition at $r$ is the same as imposing a fixed proper length $\beta$. To see this, 
we define the local temperature $T$ as

$$
T=\frac{1}{\beta},
$$

so that $\beta$ is the inverse temperature. Then, from $\beta=$ $\beta_{\infty} \sqrt{1-\frac{r_{+}^{2}}{r^{2}}}$ one finds for $T$ that $T=\frac{T_{\infty}}{\sqrt{1-\frac{r_{+}^{2}}{r^{2}}}}$, which is the Tolman temperature formula for the system formed by the black hole and cavity at $r$. This states that the temperature at the cavity is the temperature of the black hole at infinity, i.e., the Hawking temperature, blueshifted to the cavity's position $r$, or conversely, the temperature of the black hole at infinity, i.e., the Hawking temperature, is the temperature $T$ at the cavity redshifted to infinity. Using $T_{\infty}=\frac{1}{\beta_{\infty}}=\frac{1}{2 \pi r_{+}}$, we can write the canonical temperature as

$$
T=\frac{1}{2 \pi r_{+} \sqrt{1-\frac{r_{+}^{2}}{r^{2}}}} .
$$

Given a temperature $T$ and a cavity radius $r$, we want to know which values of black hole horizon radii $r_{+}$are allowed, i.e., we need to know $r_{+}=r_{+}(\beta, r)$ or $r_{+}=$ $r_{+}(T, r)$. Note that we exchange $T$ and $\beta$ at will. For that we solve the Tolman condition for temperature variation in curved space, which is given by Eq. (4). Equation (4) can be rewritten as

$$
(2 \pi T)^{2} r_{+}^{4}-(2 \pi T)^{2} r^{2} r_{+}^{2}+r^{2}=0 .
$$

Equation (5) is a quadratic equation for $r_{+}^{2}$ and can be solved exactly. In York's $d=4$ case, it is a cubic equation. Equation (5) only has real solutions for

$$
\pi r T \geq 1 .
$$

If we fix the radius $r$ of the cavity, then a black hole can form only if the temperature $T$ is high enough; otherwise, one has empty hot flat space, which if one performs a quantum treatment means hot gravitons wandering around in flat space. On the other hand, if we fix $T$ at the cavity, then only if the radius is high enough can a black hole develop; otherwise, for low $r$ one has only empty hot flat space. Equation (6) is a necessary condition for having black holes, but not sufficient.

The two possible black hole solutions $r_{+1}$ and $r_{+2}$ of Eq. (5) are given by

$$
\begin{aligned}
& r_{+1}=r\left(\frac{1-\sqrt{1-(\pi r T)^{-2}}}{2}\right)^{1 / 2}, \\
& r_{+2}=r\left(\frac{1+\sqrt{1-(\pi r T)^{-2}}}{2}\right)^{1 / 2},
\end{aligned}
$$

with $r_{+2} \geq r_{+1}$. For a connection to four dimensions, see the Appendix. Equations (7) and (8) give $r_{+}=r_{+}(r, \beta)$ or $r_{+}=r_{+}(r, T)$ for each black hole, i.e., they give the horizon radius of each black hole as a function of the cavity radius $r$ and either the inverse temperature $\beta$ or temperature $T$. In Fig. 1 1 we plot $r_{+1}$ and $r_{+2}$ as functions

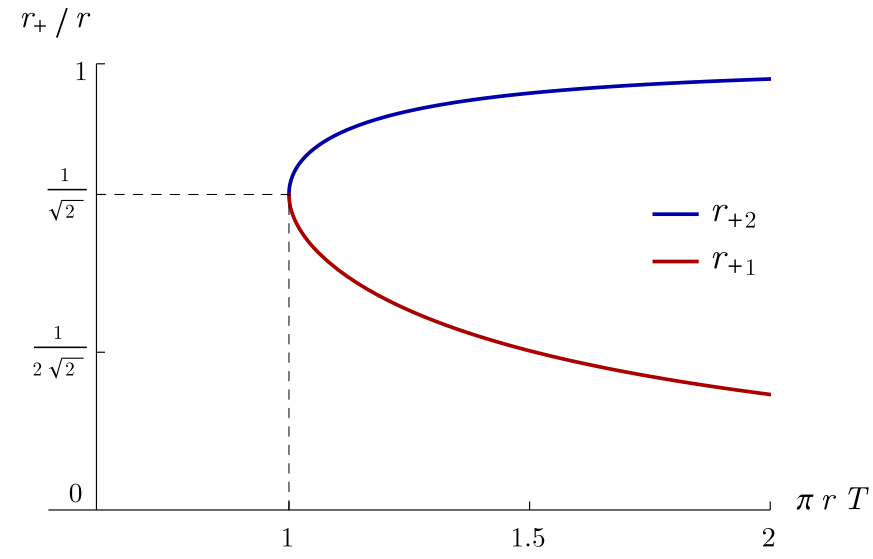

FIG. 1: The two black hole solutions $r_{+1}$ and $r_{+2}$ are represented for different parameter values of $\pi r T \geq 1$. The two coincide for $\pi r T=1$ at $\frac{r_{+}}{r}=\frac{1}{\sqrt{2}}$, represented by a point at the intersection of the dashed lines. The radius $r$ given by $\frac{r_{+}}{r}=\frac{1}{\sqrt{2}}$ is the radius of the photon sphere $r_{\mathrm{ph}}=\sqrt{2} r_{+}$. It turns out that the $r_{+1}$ solutions are thermodynamically unstable and the $r_{+2}$ solutions are thermodynamically stable.

of $T$, given in Eqs. (7) and (8). Note from Eqs. (7) and (8), and also from Fig. 1, that for low temperatures, i.e., for $\pi r T=1$ or near this value, we have $r_{+1}=r_{+2}=\frac{r}{\sqrt{2}}$. For high temperatures, as $\pi r T \rightarrow \infty$, from Eqs. (7) and 8 one has $r_{+1}=r\left(\frac{1}{2 \pi r T}+\mathcal{O}\left(\frac{1}{(\pi r T)^{3}}\right)\right)$, and $r_{+2}=r\left(1-\frac{1}{8(\pi r T)^{2}}+\mathcal{O}\left(\frac{1}{(\pi r T)^{4}}\right)\right)$, so that in the limit the smaller black hole is given by $r_{+1}=0$, while the larger one approaches the cavity's boundary $r_{+2}=r$. The radii $r_{+1}$ and $r_{+2}$ in Eqs. (7) and (8) are the radii that we need to put into the action to get the final action $I=I(r, \beta)$, to which we now turn.

\section{B. The Euclidean-Einstein action and the action functional or the partition function for a five-dimensional Schwarzschild black hole}

In the Hartle-Hawking path-integral approach to quantum gravity, the partition function $Z$ for the gravitational field is given by the integral of the Euclidean Einstein action $I$ over the space of metrics g, i.e., $Z=\int d[\mathrm{~g}] \mathrm{e}^{-\mathrm{I}[\mathrm{g}]}$. Assuming that the leading term is that of the first-order classical Euclidean action of a black hole, $I_{\mathrm{BH}}$, one has

$$
Z=\mathrm{e}^{-I_{\mathrm{BH}}} .
$$

The Euclidean Einstein action for a five-dimensional spacetime on a compact region $\mathcal{M}$ is given by

$$
I=-\frac{1}{16 \pi} \int_{\mathcal{M}} d^{5} x \sqrt{|g|} R-\frac{1}{8 \pi} \int_{\partial M} d^{4} x \sqrt{|h|}[K],
$$

where $|g|$ is the determinant of the metric $g_{a b}$, with $a, b$ running through one time and four spatial coordinates, $R$ is the Ricci scalar, $|h|$ is the determinant of the induced metric on the four-dimensional boundary $\partial \mathcal{M}$, and $[K]$ is the difference between the trace of the extrinsic curvature $K$ of the hypersurface in the metric $g$ and the extrinsic 
curvature of the hypersurface in a reference spacetime metric, which here is the Minkowski spacetime metric, $K_{\text {flat }}$. This subtraction is responsible for setting the action to zero in the reference flat spacetime. The first part of the action 10 is the bulk term, and the second part is the Gibbons-Hawking-York boundary term.

Now, for the metric Eq. (1) the Ricci scalar vanishes everywhere for the Schwarzschild metric, $R=0$, and so the first term in the action of Eq. 10 vanishes. The action then reduces to the Gibbons-HawkingYork boundary term $I=-\frac{1}{8 \pi} \int_{\partial \mathcal{M}} d^{4} x \sqrt{|h|}[K]$. The induced line element on $r=$ constant from Eq. (1) is $\left.d s^{2}\right|_{r}=\left(1-\frac{r_{+}^{2}}{r^{2}}\right) d t^{2}+r^{2} d \Omega_{3}^{2}$, the determinant $h$ of the induced metric is $h=\left(1-\frac{r_{+}^{2}}{r^{2}}\right) r^{6} \sin ^{4} \theta_{1} \sin ^{2} \theta_{2}$, and the trace of the boundary's extrinsic curvature is $K=\frac{3 \sqrt{1-\frac{r_{+}^{2}}{r^{2}}}}{r}+\frac{r_{+}^{2}}{r^{3} \sqrt{1-\frac{r_{+}^{2}}{r^{2}}}}$, while its flat-space counterpart can be found directly by putting $r_{+}=0, K_{\text {flat }}=\frac{3}{r}$. Since at the boundary $r$ is constant, one only has to integrate over the angles, which gives $2 \pi^{2}$, and integrate over $t$ from 0 to $\beta_{\infty}$. The action $I$, Eq. 10 , takes the final form

$$
I=\pi^{2} r_{+}^{3}-\frac{3}{2} \pi^{2} r_{+} r^{2}+\frac{3 \pi^{2}}{2} r_{+} \sqrt{1-\frac{r_{+}^{2}}{r^{2}}} r^{2},
$$

so that, in this form, $I=I\left(r, r_{+}\right)$.

We are interested in writing the action (11) as a function of $r$ and $\beta$ only, $I=I(r, \beta)$. This can indeed be done since $r_{+}=r_{+}(r, \beta)$, and Eq. 11] is then formally

$$
I(r, \beta)=\pi^{2} r_{+}^{3}(r, \beta)-\frac{3}{2} \pi^{2} r_{+}(r, \beta) r^{2}+\frac{3 \pi}{4} \beta r^{2},
$$

where $r_{+}$stands for $r_{+1}$ or $r_{+2}$ given in Eqs. (7) and (8). Thus, explicitly,

$$
\begin{aligned}
I\left(r, r_{+1}(r, \beta)\right) & =\frac{3 \pi^{2} r^{3}\left[(\pi r T)^{-1}-\right.}{4} \\
& \left.\left.\frac{2 \sqrt{2} \sqrt{1-\sqrt{1-(\pi r T)^{-2}}} \times}{3} \sqrt{2}\right)\right], 0 \leq \frac{r_{+1}}{r} \leq \frac{1}{\sqrt{2}},
\end{aligned}
$$

and

$$
\begin{aligned}
& I\left(r, r_{+2}(r, \beta)\right)=\frac{3 \pi^{2} r^{3}}{4}\left[(\pi r T)^{-1}-\right. \\
& -\frac{2 \sqrt{2}}{3} \sqrt{1+\sqrt{1-(\pi r T)^{-2}}} \times \\
& \left.\left(1-\frac{\sqrt{1-(\pi r T)^{-2}}}{2}\right)\right], \frac{1}{\sqrt{2}} \leq \frac{r_{+2}}{r} \leq 1
\end{aligned}
$$

where $T=\frac{1}{\beta}$, see Eq. 3 . To have black hole solutions, Eq. (6) must hold, i.e., $\pi r T \geq 1$. For the smaller black hole $r_{+}=r_{+1}$, Eq. (7), the action (13) is always positive. For the larger black hole $r_{+}=r_{+2}$, Eq. (8), the action 14 is positive for $\pi r T<\frac{2}{\sqrt{3}}$, i.e., $\pi r T<1.155$ in round numbers, or equivalently, for $\frac{r_{+}}{r}<\frac{\sqrt{3}}{2}$, i.e., $\frac{r_{+}}{r}<0.866$ in round numbers, in brief, the action exists and is positive for $1<\frac{r}{r_{+}}<\frac{2}{\sqrt{3}}$. The action 14 is zero or negative otherwise, namely,

$$
\frac{r}{r_{+}} \geq \frac{2}{\sqrt{3}}
$$

Note that $\frac{2}{\sqrt{3}}$ is the Buchdahl bound in five dimensions. In York's thermodynamic analysis in four dimensions [1] the equivalent of Eq. 15 is $\frac{r}{r_{+}} \geq \frac{9}{8}$, and $\frac{9}{8}$ is precisely the Buchdahl bound in four dimensions, a fact that went unnoticed up to now. The Buchdahl bound is a lower bound for the ratio of a star radius $r$ to its gravitational radius $r_{+}$, that arises in order to maintain the star solution free of singularities. In four dimensions the bound is given by $\frac{r}{r_{+}} \geq \frac{9}{8}$ [16], such that by defining a four-dimensional Buchdahl limiting radius $r_{\text {Buch }}=\frac{9}{8} r_{+}$one has $r \geq r_{\text {Buch }}$, whereas in five dimensions the bound is given by $\frac{r}{r_{+}} \geq \frac{2}{\sqrt{3}}$ [17], such that by defining a five-dimensional Buchdahl limiting radius $r_{\text {Buch }}=\frac{2}{\sqrt{3}} r_{+}$one has $r \geq r_{\text {Buch }}$. One sees that the Buchdahl limiting radius appears in two different contexts, namely, in the original context of star solutions and gravitational collapse in general relativity, and in the new thermodynamic context of having a negative action and thus a negative energy for a black hole in a canonical ensemble in general relativity.

\section{THERMODYNAMICS}

To study thermodynamics in the canonical ensemble of a black hole inside a cavity at temperature $T$, we connect the action $I$ with the relevant thermodynamic potential, namely, the free energy $F$. The connection is given by

$$
I=\beta F .
$$

The action $I$ is taken as Eq. (11) or in Eq. (12). On the other hand, in thermodynamics the free energy $F$ is related to the thermodynamic energy $E$, the temperature $T$, and the entropy $S$ by

$$
F=E-T S
$$

The first law of thermodynamics can be written then as

$$
d E=T d S-p d A
$$

where $A$ is the area of the cavity and $p$ is the tangential pressure at the cavity radius. The term $p d A$ is the correct term to use in the first law, rather than a volume term, one of the reasons being that the inside of the black hole has no well-defined volume in this setting, see [1. So $A$ is indeed the independent variable and the tangential pressure $p$ is conjugate to it. The first law as written in Eq. (18) envisages the energy $E$ as a function of the independent variables $S$ and $A$, i.e., $E=E(S, A)$, and $T$ 
and $p$ are state functions that in general must be provided or somehow worked out, $T=T(S, A)$ and $p=p(S, A)$, i.e., they are the equations of state. It is also useful to give the first law in a different set of variables. Note from Eq. (16) that $d I=\beta d F+F d \beta$, and using Eq. (17) for $d F$ one finds that the first law in Eq. (18) can also be written as

$$
d I=E d \beta-p d A
$$

where here $I$ is taken as a function of the independent variables $\beta$ and $A, I=I(A, \beta)$. In this form one finds immediately that

$$
\begin{aligned}
E & =\left(\frac{\partial I}{\partial \beta}\right)_{A}, \\
p & =-\frac{1}{\beta}\left(\frac{\partial I}{\partial A}\right)_{\beta}, \\
S & =\beta E-I .
\end{aligned}
$$

Note also that $I$ can be seen as a function of the independent variables $\beta$ and $r, I=I(r, \beta)$, instead of $\beta$ and $A, I=I(A, \beta)$, since a three-dimensional sphere has area $A$ given by

$$
A=2 \pi^{2} r^{3}
$$

Let us now find $E, p$, and $S$ for the system.

To find $E$ we have to work out $\left(\frac{\partial I}{\partial \beta}\right)_{A}$, and we proceed as follows. Envisaging $I=I(r, \beta)$, one has $d I=\left(\frac{\partial I}{\partial \beta}\right)_{r} d \beta+\left(\frac{\partial I}{\partial r}\right)_{\beta} d r$. Envisaging $I=I\left(r, r_{+}\right)$, one has $d I=\left(\frac{\partial I}{\partial r_{+}}\right)_{r} d r_{+}+\left(\frac{\partial I}{\partial r}\right)_{r_{+}} d r$. Both $d I \mathrm{~s}$ are the same, and so at constant $r$ we can write $\left(\frac{\partial I}{\partial \beta}\right)_{r}=\frac{\left(\partial I / \partial r_{+}\right)_{r}}{\left(\partial \beta / \partial r_{+}\right)_{r}}$. From Eq. 11 we have $\left(\frac{\partial I}{\partial r_{+}}\right)_{r}=$ $\frac{3 \pi^{2} r^{2}\left(1-\sqrt{1-r_{+}^{2} / r^{2}}\right)}{2 \sqrt{1-r_{+}^{2} / r^{2}}}\left(1-\frac{2 r_{+}^{2}}{r^{2}}\right)$. From Eq. (2) we get $\left(\frac{\partial \beta}{\partial r_{+}}\right)_{r}=\frac{2 \pi}{\sqrt{1-r_{+}^{2} / r^{2}}}\left(1-\frac{2 r_{+}^{2}}{r^{2}}\right)$. Then, from Eq. 20 we get the thermodynamic energy $E$,

$$
E=\frac{3 \pi r^{2}}{4}\left(1-\sqrt{1-\frac{r_{+}^{2}}{r^{2}}}\right)
$$

It is interesting to note that the total thermodynamic energy $E$ inside the cavity is actually larger than the spacetime's ADM mass. Indeed, using $r_{+}^{2}=\frac{8}{3 \pi} m$ together with Eq. 24 , one finds $m=E-\frac{2 E^{2}}{3 \pi r^{2}}$, so that the spacetime's total energy is read as the thermodynamic energy inside the cavity plus its binding energy.

To find the pressure from Eq. 21 we use $\left(\frac{\partial I}{\partial r}\right)_{\beta}=$ $\left(\frac{\partial I}{\partial r}\right)_{r_{+}}-\left(\frac{\partial I}{\partial \beta}\right)_{r}\left(\frac{\partial \beta}{\partial r}\right)_{r_{+}}$, together with Eqs. 22 and 12. This gives

$$
p=\frac{1}{8 \pi r \sqrt{1-\frac{r_{+}^{2}}{r^{2}}}}\left(1-\sqrt{1-\frac{r_{+}^{2}}{r^{2}}}\right)^{2} .
$$

Now we can find the entropy $S$ of the system. From Eq. 22, together with Eqs. 12 and 24 , we find that the total entropy inside the cavity becomes $S=\pi^{2} r_{+}^{3} / 2$, i.e.,

$$
S=\frac{A_{+}}{4}
$$

which is the Bekenstein-Hawking area law for the black hole entropy, with the five-dimensional surface area of the black hole given by Eq. (23) putting $r=r_{+}$, i.e., $A_{+}=2 \pi^{2} r_{+}^{3}$. Here $r_{+}$can be either $r_{+1}$ or $r_{+2}$. Knowing $S$, one can again find $p$ using instead the first law as written in Eq. 18. Then, $p=-\left(\frac{\partial E}{\partial A}\right)_{S}$ and so $p=\frac{1}{8 \pi r \sqrt{1-\frac{r_{+}^{2}}{r^{2}}}}\left(1-\sqrt{1-\frac{r_{+}^{2}}{r^{2}}}\right)^{2}$, which coincides with Eq. 25), and where Eq. 24) has been used.

One can also work out the Euler relation, the scaling laws, and the Gibbs-Duhem relation. From the energy and entropy in Eqs. (24) and (26), it is straightforward that $E=\frac{3 \pi}{4}\left(\frac{A}{2 \pi^{2}}\right)^{2 / 3}\left(1-\sqrt{1-\left(\frac{4 S}{A}\right)^{2 / 3}}\right)$. Using Euler's theorem for homogeneous functions, one has that $E$ is homogeneous of degree $\frac{2}{3}$ in $A$ and $S$, i.e., $\frac{2}{3} E=\left(\frac{\partial E}{\partial S}\right) S+\left(\frac{\partial E}{\partial A}\right) A$, and the Euler relation for the cavity is obtained as

$$
\frac{2}{3} E=T S-p A
$$

The scaling laws for the canonical ensemble are thus $r \rightarrow \lambda r\left(A \rightarrow \lambda^{3} A\right), T \rightarrow \lambda^{-1} T(\beta \rightarrow \lambda \beta), E \rightarrow \lambda^{2} E$, and $S \rightarrow \lambda^{3} S$. It makes sense that the temperature is no longer an intensive parameter, due to the fact that in gravitational systems thermodynamic equilibrium is obtained through Tolman's formula for the redshifted temperature. The same goes for the pressure, where with Eq. 27 one finds that the pressure scales as $p \rightarrow \lambda^{-1} p$. Also, the action $I$ and free energy $F$ scale as $I \rightarrow \lambda^{3} I$ and $F \rightarrow \lambda^{2} F$. Taking the differential of the Euler relation and using the first law, the Gibbs-Duhem relation is

$$
3 S d T+d E-3 A d p=0
$$

\section{THERMAL STABILITY}

Thermal stability in the canonical ensemble is determined by the heat capacity at constant cavity area, $C_{A}$, which has to be equal to or greater than zero, $C_{A} \geq 0$. $C_{A}$ is defined by

$$
C_{A} \equiv\left(\frac{\partial E}{\partial T}\right)_{A}
$$

$E$ is given by Eq. (24) in the form $E=E\left(r_{+}, r\right)$, with $r_{+}=r_{+}(\beta, r)$ given in Eq. (2). Now, $A=$ const means $r=$ const, and since $T=1 / \beta$, see Eq. (3), we have $C_{A} \equiv\left(\frac{\partial E}{\partial T}\right)_{A}=-\beta^{2} \frac{\left(\partial E / \partial r_{+}\right)_{r}}{\left(\partial \beta / \partial r_{+}\right)_{r}}$. Then, from Eq. 24 we find that $\left(\frac{\partial E}{\partial r_{+}}\right)_{r}=\frac{3 \pi}{4 \sqrt{1-r_{+}^{2} / r^{2}}} r_{+}$, and from Eq. 2 we have $\left(\frac{\partial \beta}{\partial r_{+}}\right)_{r}=\frac{2 \pi}{\sqrt{1-r_{+}^{2} / r^{2}}}\left(1-\frac{2 r_{+}^{2}}{r^{2}}\right)$. So, the heat 
capacity is given by

$$
C_{A}=\frac{3 \pi^{2} r_{+}^{3}}{2} \frac{\left(1-\frac{r_{+}^{2}}{r^{2}}\right)}{\left(\frac{2 r_{+}^{2}}{r^{2}}-1\right)} .
$$

Thus. thermal stability,

$$
C_{A} \geq 0,
$$

means that the cavity's boundary must be located between the horizon radius and the photon sphere radius, $r_{\mathrm{ph}}$,

$$
r_{+} \leq r \leq r_{\mathrm{ph}},
$$

where in five dimensions $r_{\mathrm{ph}}=\sqrt{2} r_{+}$[18]. The same type of result appears in York's four-dimensional treatment, i.e., for $r_{+} \leq r \leq r_{\mathrm{ph}}$ with $r_{\mathrm{ph}}=\frac{3}{2} r_{+}$in $d=4$, the heat capacity is positive and the canonical system is thermodynamically stable. Thus, it is clear that the the photon sphere plays an intrinsic role in thermodynamic stability, since it appears consistently as a bound in both four and five dimensions, see also [11. In addition, Eqs. 30 - $(32)$ imply that the black hole with horizon radius $r_{+1}$ given in Eq. (7) is always thermodynamically unstable and the black hole with horizon radius $r_{+2}$ given in Eq. (8) is always thermodynamically stable.

\section{FREE ENERGY FUNCTION OF THE FIVE-DIMENSIONAL BLACK HOLE: THERMODYNAMIC INTERPRETATION OF THE PHYSICAL PROCESSES}

We can make progress and understand the physical processes and thermodynamic instabilities further within the thermodynamic approach using a generalized free energy.

The free energy $F$ in our problem was defined for $r_{+}=$ $r_{+1}$ and $r_{+}=r_{+2}$, i.e., $F=F\left(r_{+}, r\right)$ or $F=F(\beta, r)$ with $r_{+}$taking those two values. It is of interest to define a generalized canonical free energy $\bar{F}$ as $\bar{F}=E-T S$ where the boundary radius $r$ and the temperature $T$ are still kept fixed, but the horizon radius $r_{+}$can assume all values, not just the two above. Using such an extension $\bar{F}$ for the five-dimensional free energy, as was done in four dimensions in [1, one can analyze radial, i.e., spherical, and static perturbations to the thermodynamic system we are studying, thus providing results for the possible stable and unstable configurations.

From Eqs. (24) and (26) we find that the extended free energy $\bar{F}=E-T S$ can be written as $\bar{F}=\bar{F}\left(r_{+}, r, T\right)$ and is given by

$$
\bar{F}\left(r_{+}, r, T\right)=\frac{3 \pi r^{2}}{4}\left(1-\sqrt{1-\frac{r_{+}^{2}}{r^{2}}}-\frac{2 \pi r T}{3} \frac{r_{+}^{3}}{r^{3}}\right) .
$$

With this $\bar{F}$ we are considering static $r_{+}$deviations from the two solutions $r_{+}=r_{+1}$ and $r_{+}=r_{+2}$. In Fig. 2 we plot $\bar{F}$ as a function of $r_{+}$, keeping $r$ and $T$ fixed, as given in Eq. (33). The extrema in the figure occur for $\left(\frac{\partial \bar{F}}{\partial r_{+}}\right)_{r, T}=0$. Solving for these extrema, one finds that they occur for $r_{+}=0, r_{+}=r_{+1}$, and $r_{+}=r_{+2}$, i.e., for no black hole, and for the two black hole solutions in the canonical ensemble. Indeed, the no black hole case $r_{+}=0$ is a solution with $\bar{F}=0$. We have been dealing with the classical gravitational action for the canonical ensemble thermodynamic approach and the no black hole case corresponds to having a boundary $r$ at temperature $T$ with nothing inside, only Minkowski, flat spacetime at temperature $T$. This is classical hot flat space, i.e., classical vacuum at temperature $T$ with zero free energy. From Fig. 2 we see that the smaller black hole at $r_{+1}$ is an unstable equilibrium point and it has $C_{A}<0$, while the larger black hole $r_{+2}$ is a stable equilibrium point and it has $C_{A}>0$, as found previously. One also finds from $\left(\frac{\partial \bar{F}}{\partial r_{+}}\right)_{r, T}=0$ that there are extrema only when $\pi r T \geq 1$, with the equality yielding the case $r_{+1}=r_{+2}$, a result consistent with our findings. Note that $\bar{F}$ in Eq. (33), see also Fig. 2, has been defined for all $r_{+}$, but for a value of $r_{+}$other than $r_{+}=0$ and the two black hole values $r_{+1}$ and $r_{+2}$ the geometry has a conical defect in the real Euclidean plane and the corresponding temperature does not correspond to a physical situation, i.e., the corresponding black hole does not have the correct temperature since it does not have a temperature compatible with the temperature $T$ of the boundary, and so it is not an equilibrium configuration.

For $\pi r T<1$ only classical hot flat space, with $r_{+}=0$, is possible inside the cavity at radius $r$, as can be deduced from Eq. (33) or seen in Fig.2, i.e., it is impossible to have black holes, see also Eq. (6). For $\pi r T \geq 1$ the black hole solutions $r_{+1}$ and $r_{+1}$ are possible, but to extract the full details a careful analysis with $\bar{F}$ has to be performed. It is useful to resort to Fig. 22 For $\pi r T \geq 1$ one can follow the possibility of forming black holes from hot flat space through a nucleation instability. Classical hot flat space has $r_{+}=0$ by definition. The smaller black hole $r_{+1}$ always has the boundary $r$ obeying $r>r_{\mathrm{ph}}$, always has positive free energy, i.e., positive action $I\left(r_{+1}\right)$ given by Eq. (13), where from now on we shorten the notation $I\left(r_{+1}\right) \equiv I\left(r, r_{+1}(r, \beta)\right)$, is at a maximum of $\bar{F}$, see Fig. 2, and this means it is unstable, with the thermal instability also being seen through the negativity of the specific heat. The larger black hole $r_{+2}$ always has the boundary $r$ that obeys $r<r_{\mathrm{ph}}$, can have both either positive or negative free energy, i.e., positive or negative action $I\left(r_{+2}\right)$ given by Eq. (14) with the notation shortened, respectively, is at a minimum of $\bar{F}$, see Fig. 2, and this means it is stable, with the thermal stability also being seen through the positivity of the specific heat. The maximum of $\bar{F}$ at $r_{+1}$ forms a potential barrier between classical hot flat space at $r_{+}=0$ and the larger stable black hole $r_{+2}$ at a minimum of $\bar{F}$ at $r_{+2}$. It is then of interest to analyze for which conditions the stable solution $r_{+2}$ can be reached through the unstable one $r_{+1}$ from hot flat space $r_{+}=0$.

It is known that in the canonical ensemble, any spontaneous process goes in the direction of decreasing $F$. In the canonical ensemble it is forbidden to increase $F$, in much the same way that it is forbidden to decrease the entropy $S$, i.e., violate the second law of thermodynam- 


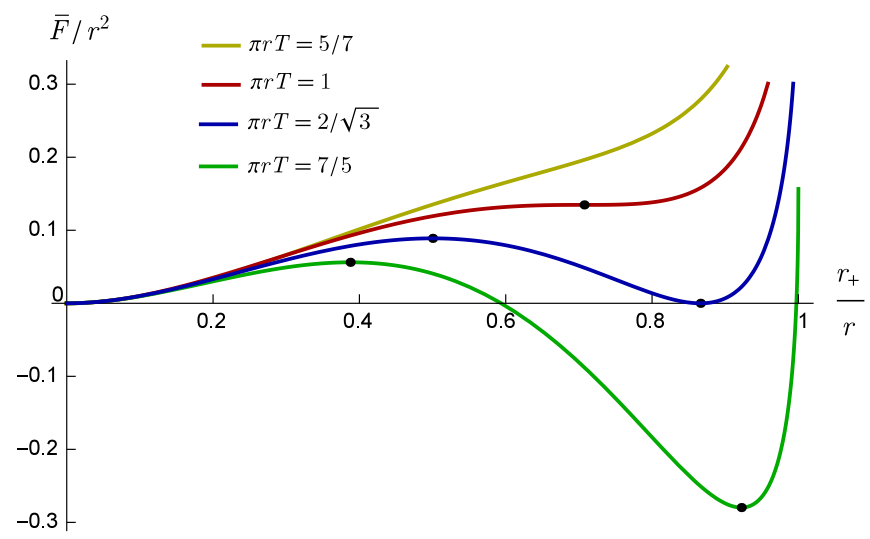

FIG. 2: The free energy function for four different situations, namely, $\pi r T=\frac{5}{7}, \pi r T=1, \pi r T=\frac{2}{\sqrt{3}}$, and $\pi r T=\frac{7}{5}$. Classical hot flat space is at $r_{+}=0$ and has zero free energy. The black hole solutions $r_{+1}$ and $r_{+2}$ occur at the extrema $\left(\frac{\partial \bar{F}}{\partial r_{+}}\right)_{r, T}=0$, indicated on the plot by the black dots. The upper yellow curve $\pi r T=\frac{5}{7}$, an instance of $\pi r T<1$, is for the case where there are no black hole solutions. The red curve is for the limiting case $\pi r T=1$, where the two black hole solutions coincide at the inflection point, $r_{+1}=r_{+2}$, resulting in a neutral equilibrium point. As $\pi r T$ increases, the smaller black hole $r_{+1}$ is an unstable equilibrium point, while the larger black hole $r_{+2}$ is stable. The smaller black hole $r_{+1}$ always has positive free energy, and the larger black hole $r_{+2}$ can have positive or negative free energy depending on the value of $\pi r T$. For $\pi r T<\frac{2}{\sqrt{3}}$ the free energy of the larger black hole $r_{+2}$ is positive, for $\pi r T=\frac{2}{\sqrt{3}}$ the free energy of the larger black hole $r_{+2}$ is zero, and for $\pi r T>\frac{2}{\sqrt{3}}$, e.g., $\pi r T=\frac{7}{5}$ the free energy of the larger black hole $r_{+2}$ is negative, and classical hot flat space $r_{+}=0$ which has zero free energy can nucleate in this latter case into the larger $r_{+2}$ black hole through the $r_{+1}$ black hole.

ics in the microcanonical ensemble, where the thermodynamic energy $E$ and the size of the system are held fixed. Thus, a phase transition in the canonical ensemble from classical hot flat space $r_{+}=0$ to the larger stable black hole $r_{+2}$ can occur as a spontaneous process if the $r_{+2}$ black hole has lower free energy than hot flat space. Classically, hot flat space has zero free energy, so if the $r_{+2}$ black hole has negative free energy nucleation can occur to a black hole $r_{+2}$. Thus, to have nucleation one requires $F\left(r_{+2}\right) \leq 0$, i.e., $I\left(r_{+2}\right) \leq 0$, where the equality holds when the two phases coexist. From Eq. (14), this yields

$$
\pi r T \geq \frac{2}{\sqrt{3}},
$$

which in round numbers is $\pi r T \geq \frac{2}{\sqrt{3}}=1.1547$. This is a necessary and sufficient condition for nucleation from classical hot flat space to the stable black hole $r_{+2}$ through the unstable black hole $r_{+1}$. Equation (34) is a stronger condition than the $\pi r T \geq 1$ condition of Eq. (6) for having black hole solutions at all. Conversely, one finds that a large black hole $r_{+2}$ decays into classical hot flat space when the condition $F\left(r_{+2}\right) \geq 0$ holds, i.e., $I\left(r_{+2}\right) \geq 0$. From Eq. 14 this yields $\pi r T \leq \frac{2}{\sqrt{3}}$, which together with the $\pi r T \geq 1$ condition for having black holes gives that a large black hole $r_{+2}$ can decay into classical hot flat space when

$$
1 \leq \pi r T \leq \frac{2}{\sqrt{3}} .
$$

Also, within this range classical hot flat space will never nucleate into a black hole.

\section{ACTION FUNCTIONAL TO SECOND ORDER AND ITS RELATION TO THERMODYNAMICS AND THERMODYNAMIC STABILITY}

In the Hartle-Hawking path-integral approach to quantum gravity [2 4, the partition function $Z$ for the gravitational field is given by the integral of the Euclidean Einstein action $I$ over the space of metrics g, i.e., $Z=$ $\int d[\mathrm{~g}] \exp (-\mathrm{I}[\mathrm{g}])$. The path-integral approach of the canonical ensemble for black holes [1] uses the classical action, i.e., the zero-order Euclidean Einstein action $I_{\mathrm{BH}}$, for the possible black hole solutions together with the corresponding partition function $Z=\exp \left(-I_{\mathrm{BH}}\right)$, giving the black hole thermodynamic properties that we have been analyzing. We have seen that for a given temperature $T$ at some cavity radius $r$, there is classical hot flat space $r_{+}=0$, and there are two black hole radii, $r_{+1}$ and $r_{+2}$, these three situations are the ones that fit the boundary data. Two important results in this formalism are a formula for the black hole entropy and that there can be a phase transition from classical hot flat space to the larger stable black hole $r_{+2}$ through the smaller unstable one $r_{+1}$ and vice versa, as a detailed analysis of the free energy has shown.

Now, a next-order quantum gravity treatment should include the fact that hot flat space, rather than being empty as we have been considered so far, contains gravitons and so its free energy is nonzero, in fact is negative. Indeed graviton radiation in thermal equilibrium in a cavity of radius $r$ at temperature $T$ is graviton blackbody radiation. In addition, a Schwarzschild black hole is also made of gravitons and to understand it in more detail one should study perturbations due to quantum thermal fluctuations around the Euclidean black hole solutions.

Thus, to proceed with the path-integral formulation and to calculate the partition function $Z=$ $\int d[\mathrm{~g}] \exp (-\mathrm{I}[\mathrm{g}])$ anew to include fluctuations around the Euclidean black hole solutions, also called black hole instantons in this context, one considers small metric perturbations $h_{a b}$ to the instanton metric $\bar{g}_{a b}$, such that $g_{a b}=\bar{g}_{a b}+h_{a b}$. The action $I$ is now $I=I[\bar{g}]+I_{2}[h, \bar{g}]$, where a first-order term does not appear because the instanton satisfies the equations of motion and terms with order higher than two are discarded. The partition function is then $Z=\mathrm{e}^{-I[\bar{g}]} \int d[h] \mathrm{e}^{-I_{2}[h, \bar{g}]}=\mathrm{e}^{-I[\bar{g}]} Z_{2}$, for some function $Z_{2}$. The perturbed part $I_{2}[h, \bar{g}]$ reveals the fluctuating modes, their eigenfunctions, and eigenvalues. There are two possibilities depending on the latter. If in the perturbation one of the modes gets a negative eigenvalue one has a negative mode around the given instanton 
and the perturbation is unstable. This in turn means that the action and thus the free energy has an imaginary part, and that the partition function also gets an imaginary exponential. Going back to the Lorentzian representation, the associated action functional has a negative exponential signaling a decaying process, i.e., an instability of the system. On the other hand, there is the possibility that all of the perturbed modes have positive eigenvalues, in this case the perturbation is stable around the given instanton.

In [5], it was found for a four-dimensional spacetime, that in the canonical ensemble, i.e., for fixed $T$ at a fixed boundary with very large radius $r$, perturbations around the instanton $r_{+1}$ yield a negative eigenvalue for which the corresponding mode decreases the action. So, the instanton is a saddle point rather than a minimum, and is unstable. For this small black hole $r_{+1}$ the free energy then gets an imaginary part which is inherited by the partition function. Then, the path integral is an exponential with a negative exponent, yielding a mode with a finite decay time, either to quantum hot flat space or to the large black hole $r_{+2}$ [5]. In [6] it was further shown in four dimensions that, modulo gauge boundary conditions, the negative mode disappears and the system is stable when the cavity radius $r$ obeys $r \leq r_{\mathrm{ph}}$, where again $r_{\mathrm{ph}}$ is the radius of the circular photon orbits of the spacetime, i.e., $r_{\mathrm{ph}}=\frac{3}{2} r_{+}$for four-dimensional Schwarzschild spacetime. This means that if one calculates the fluctuations around the instanton $r_{+2}$ one finds that the action has no negative eigenvalue and the modes increase the action away from the instanton; thus it has a minimum, the partition function is real, there are no decaying modes, and the black hole is stable. Such a perturbation analysis was done in [11 in five and higher dimensions for the two Schwarzschild instantons $r_{+1}$ and $r_{+2}$, and it was indeed found that for a cavity radius $r$ less than or equal to a given radius the negative mode disappears. This radius is the radius of the circular photon orbits, $r_{\mathrm{ph}}$, which in five dimensions is $r_{\mathrm{ph}}=\sqrt{2} r_{+}$.

Clearly, one expects the instability provided by the heat capacity and the free energy thermodynamic phase transition analysis done previously to be related to the action functional path integral instability and the corresponding phase transitions. This is indeed the case. From the thermodynamic point of view, the small black hole solution $r_{+1}$ is a maximum of the free energy function and has negative heat capacity, which means that it is an unstable solution. From the second-order action functional path-integral analysis, the black hole instanton $r_{+1}$ is a saddle point, as it has a negative eigenvalue that decreases the action, rendering the solution unstable. It acts as a barrier over which hot gravitons can be excited. From the thermodynamic point of view, the large black hole solution $r_{+2}$ is a minimum of the free energy function and has positive heat capacity, which means that it is a stable solution. From the second-order action functional path-integral analysis, the black hole instanton $r_{+2}$ is a minimum of the action, it has no negative eigenvalues, and the solution is stable. Both points of view show that hot flat space is metastable in some circumstances and can transition and nucleate the large black hole $r_{+2}$ through the barrier provided by the small black hole $r_{+1}$. The definite connection between the thermodynamic heat capacity and the saddle approximation of the action, i.e., that the specific heat of the black hole becomes positive when the negative mode of the black hole instanton disappears, was made clearer in [12] where a connection to a classical mechanical instability of black branes was also made.

\section{GROUND STATE OF THE CANONICAL ENSEMBLE: QUANTUM HOT FLAT SPACE, BLACK HOLE, OR BOTH}

The action functional perturbation analysis has thus showed that in order to properly understand the physics involving black holes one has to treat hot flat space in quantum terms rather than in classical terms. Knowing this, one can address the important issue of determining the ground state of the canonical ensemble. By definition, the ground state of the canonical ensemble is the one with the lowest free energy $F$ or, since $I=\beta F$, the one with the lowest action $I$. In the canonical ensemble for hot gravity that we are studying, there are three possible phases: quantum hot flat space, the large $r_{+2}$ black hole, or a superposition of these two phases. Thus, to decide which one is the ground state we have to compare the free energy of quantum hot flat space $F_{\text {HFS }}$ with the free energy $F\left(r_{+2}\right)$ for the stable black hole $r_{+2}$.

First, we determine the free energy of quantum hot flat space. In quantum hot flat space one treats the system as a quantum gas of gravitons living in a cavity of radius $r$ at temperature $T$. Thus, quantum hot flat space is a blackbody of gravitons at a given temperature. The internal energy of free gravitons inside a cavity of a four-dimensional fixed spatial four-volume $V$ of arbitrary shape and fixed temperature $T$ is $E_{\mathrm{HFS}}=5 \mathrm{aVT} T^{5}$, where the 5 in front of the expression is the total number of degrees of freedom of a massless graviton in five dimensions, and $a$ is a number given by $a=\frac{3 \zeta(5)}{\pi^{2}}$, where $\zeta$ is the Riemann zeta function with $\zeta(5)=1.03693$ in round numbers, and it is related to the Stefan-Boltzmann constant in four spatial dimensions $\sigma$ through $a=\frac{8}{3 \pi} \sigma[19]$. A spherical cavity of four spatial dimensions has volume $V=\frac{\pi^{2} r^{4}}{2}$ and the equation of state for radiation is $E_{\mathrm{HFS}}=4 P_{\mathrm{HFS}} V$, where $P_{\mathrm{HFS}}$ is the radiation pressure given by $P_{\mathrm{HFS}}=\frac{5}{4} a T^{5}$. Then, the entropy $S_{\mathrm{HFS}}$ follows from the first law of thermodynamics applied to the radiation in the cavity, $T d S_{\mathrm{HFS}}=d E_{\mathrm{HFS}}+P_{\mathrm{HFS}} d V$, i.e., $S_{\mathrm{HFS}}=\frac{3 E_{\mathrm{HFS}}}{4 T}$. The free energy of quantum hot flat space can now be calculated from $F_{\mathrm{HFS}}=E_{\mathrm{HFS}}-T S_{\mathrm{HFS}}$ and it is

$$
F_{\mathrm{HFS}}=-\frac{5 a \pi^{2}}{8} r^{4} T^{5} .
$$

Quantum hot flat space has a free energy with a $r^{4} T^{5}$ dependence, and it is negative, rather than zero as in the classical approximation. In the case where one wants to work with the action for quantum hot flat space, one has $I=\beta F$ with $\beta=1 / T$, and so $I_{\mathrm{HFS}}=-\frac{5 \pi^{2} a}{8} r^{4} T^{4}$.

Second, the free energy $F\left(r_{+2}\right)$ for the stable black hole $r_{+2}$ is given by $I\left(r_{+2}\right)$ of Eq. 14, where again we have 
shortened the notation. Since $I=\beta F$, one gets

$$
\begin{aligned}
& F\left(r_{+2}\right)=\frac{3 \pi^{2} r^{3} T}{4}\left[(\pi r T)^{-1}-\right. \\
& \left.-\frac{2 \sqrt{2}}{3} \sqrt{1+\sqrt{1-(\pi r T)^{-2}}}\left(1-\frac{\sqrt{1-(\pi r T)^{-2}}}{2}\right)\right] .
\end{aligned}
$$

Now one has to directly compare $F_{\mathrm{HFS}}$ given in Eq. (36) with $F\left(r_{+2}\right)$ given in Eq. (37). Comparing both free energies, one has that the stable black hole is the ground state whenever

$$
F\left(r_{+2}\right) \leq F_{\mathrm{HFS}},
$$

with the equality holding when both phases coexist.

Using Eqs. (36) and (37) in Eq. (38) we find

$$
\begin{aligned}
& \frac{5 \pi^{2} a}{8} r^{-3}<-\frac{3 \pi^{3}}{4}(\pi r T)^{-4}\left[(\pi r T)^{-1}-\right. \\
& \left.-\frac{2 \sqrt{2}}{3} \sqrt{1+\sqrt{1-(\pi r T)^{-2}}}\left(1-\frac{\sqrt{1-(\pi r T)^{-2}}}{2}\right)\right]
\end{aligned}
$$

The right-hand side of Eq. (39) has a maximum with respect to $\pi r T$ at $\pi r T=\frac{121}{8 \sqrt{105}}$, i.e., $\pi r T=1.47605$ in round numbers. For that maximum, again from Eq. (39), there is a corresponding critical radius $r_{c}$ given by $r_{c}=\frac{1331}{336} \sqrt[3]{\frac{121 a}{20 \sqrt{105} \pi^{4}}}$, i.e., $r_{c}=0.491529$ in round numbers. If the radius is higher than the critical value $r_{c}$ then the black hole is the ground state for some cavity temperature $T$, if the radius is lower than the critical value $r_{c}$ then the ground state is quantum hot flat space, and if there is equality the ground state is made of both phases living together, see Fig. 3 .

We can also use Eq. (38) to find a critical cavity temperature $T_{c}$. Indeed, using again Eqs. (36) and (37), Eq. 38 can also be written as

$$
\begin{aligned}
& \frac{5 \pi^{2} a}{8} T^{3} \leq-\frac{3 \pi^{3}}{4}(\pi r T)^{-1}\left[(\pi r T)^{-1}-\right. \\
& \left.-\frac{2 \sqrt{2}}{3} \sqrt{1+\sqrt{1-(\pi r T)^{-2}}}\left(1-\frac{\sqrt{1-(\pi r T)^{-2}}}{2}\right)\right] .
\end{aligned}
$$

The right-hand side of Eq. 40 has a maximum with respect to $\pi r T$ at $\pi r T=\frac{25}{4 \sqrt{6}}$, in round numbers this is $\pi r T=2.55155$. For that maximum, again from Eq. 40, there is a corresponding critical temperature $T_{c}$ given by $T_{c}=\frac{4}{5} \sqrt[3]{\frac{36 \pi}{125 a}}$, which with $a=0.315188$ approximately, gives $T_{c}=1.13697$ in round numbers. If the temperature is lower than the critical value $T_{c}$ then the black hole is the ground state for some cavity radii $r$, if the temperature is higher than the critical value $T_{c}$ then the ground state is quantum hot flat space made of gravitons, and if the equality holds the ground state is both phases living together, see Fig. 3 .

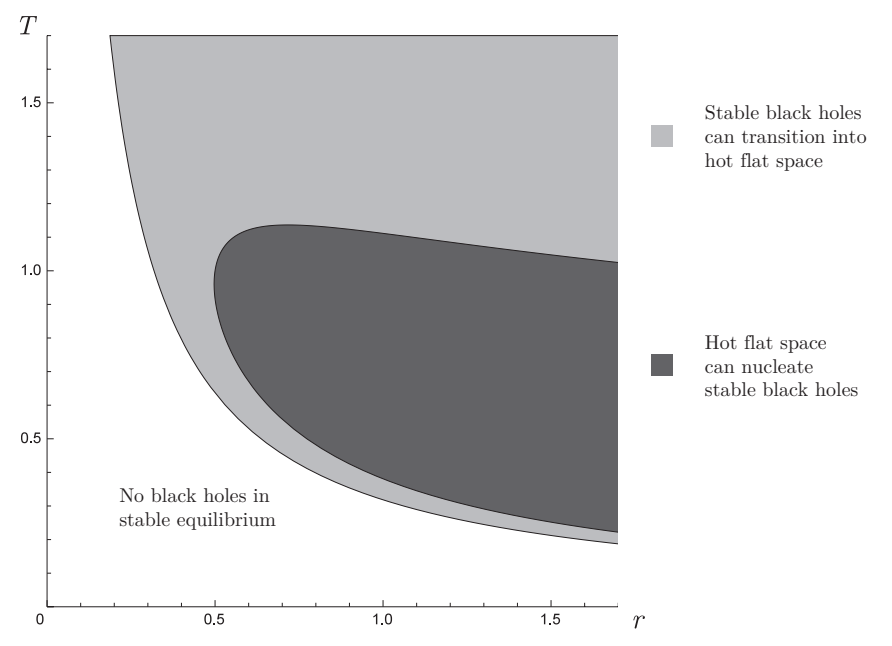

FIG. 3: In the $(r, T)$ plot, each configuration of the canonical ensemble is represented by a different point. The white region defined by $\pi r T<1$, see Eq. (6), is the region where black holes are not possible. The gray region has quantum hot flat space as the ground state. The black region has the larger stable black hole $r_{+2}$ as the ground state of the canonical ensemble. The thick black line corresponds to the situation where the ground state is a superposition of the black hole state and quantum hot flat space. These regions are separated by the line implicitly defined in Eq. (38). It is interesting to comment on the hyperbola $\pi r T=\frac{2}{\sqrt{3}}$ that appears in Eq. (34). This line is not drawn so as not to confuse the figure. Above this hyperbola the black hole action is negative and below it the black hole action is positive. The hyperbola appears naturally when one considers classical hot flat space with zero free energy rather than quantum hot flat space with negative free energy. One finds that for relatively large $r$, i.e., $r \gtrsim 1.0$ this classical line coincides with the thick black line of the figure, which is the line of coexistence, so classical hot flat space is a good approximation in this region. For relatively small $r \lesssim 1.0$ the classical line hyperbola detaches from the black line of coexistence, meaning the quantum theory for hot flat space is needed in the region of small $r$ and high $T$.

In brief, the canonical ensemble for hot gravity in five dimensions possesses a ground state that can be either a quantum hot flat space phase, a large black hole phase, or a superposition of these two phases. Which phase dominates is determined by the cavity radius $r$ and temperature $T$, as shown in Fig. 3. If one changes the cavity radius or temperature, a phase transition from one phase to the other might occur. It is worth noting that the phase transition implies a change of topology of space. For instance, when the phase changes from quantum hot flat space to a black hole the topology changes from $R^{5}$ of flat space to $R^{2} \times S^{3}$ of the Euclidean black hole. Now, the Euler characteristic is a measure of the topology of the space. In the transition from classical hot flat space $r_{+}=0$ to the stable $r_{+2}$ black hole through the $r_{+1}$ black hole, the Euler characteristic $\chi$ of the Euclidean $(t, r)$ disk 14. of the sequence of geometries takes the value $\chi=0$ for classical flat space at $r_{+}=0$ and the value $\chi=1$ at $r_{+1}$ and $r_{+2}$. The Euler characteristic is not defined for other values of $r_{+}$. Therefore, in the transition there is a change of spatial topology, a fact that is known to 
be possible in quantum gravity at finite temperature but impossible in classical gravity. It is also interesting to note that we have found that in the Planckian regime, namely, $r=1$ and $T=1$ the black hole is the ground state of the ensemble. This result is altered if quantum hot flat space has many additional fundamental species on top of the five graviton degrees of freedom; in such a case, the ground state at the Planck scale is quantum hot flat space.

\section{DENSITY OF STATES}

We now show that by using the partition function representation as a sum over energies $E$ of the density of states $\nu$ weighted with the Boltzmann factor $e^{-\beta E}$, one can verify that $\nu$ is proportional to the logarithm of $\frac{A_{+2}}{4}$, establishing that the entropy of the $r_{+2}$ black hole is indeed $S=\frac{A_{+2}}{4}$.

In the canonical ensemble, the partition function $Z(\beta, r)$ can be written as an integral over the energies of the system in the form $Z(\beta, r)=\int d E \nu(E, r) e^{-\beta E}$, with $\nu(E, r)$ being the density of states at given $E$ and cavity radius $r$. Conversely, given a partition function $Z(\beta, r)$ one can calculate for a given $r$ the number of states $\nu(E, r) d E$ between $E$ and $E+d E$ using the Laplace transform $\nu(E, r)=\frac{1}{2 \pi i} \int_{-i \infty}^{i \infty} d \beta Z(\beta, r) e^{\beta E}$. For the stable black hole $r_{+2}$, the partition function reads $Z=e^{-I\left(r_{+2}\right)}$, where $I\left(r_{+2}\right)$ is given in Eq. (14). Since for the existence of black holes one has $\pi r T>1$, see Eq. (6), we can approximate $r_{+2}$, see Eq. (8), to second order in $1 /(\pi r T)^{2}$ giving $r_{+2}=r\left(1-\frac{1}{8(\pi r T)^{2}}+\mathcal{O}\left(T^{-4}\right)\right)$, and then with $I\left(r_{+2}\right)$, see Eq. 14 , to second order in $1 /(\pi r T)^{2}$ we can write the partition function (9) as

$$
Z(\beta, r) \simeq \exp \left(\frac{\pi^{2}}{2} r^{3}-\frac{3 \pi}{4} \beta r^{2}+\frac{3}{16} \beta^{2} r\right) .
$$

Putting this into the Laplace transform $\nu(E, r)=$ $\frac{1}{2 \pi i} \int_{-i \infty}^{i \infty} d \beta Z(\beta, r) e^{\beta E}$ one finds that the density of states is $\nu(E)=\frac{2}{\sqrt{3 \pi r}} \exp \left(\frac{\pi^{2} r^{3}}{2}-\frac{4}{3 r}\left(E-\frac{3 \pi r^{2}}{4}\right)^{2}\right)$. Using from Eq. 24 that the ADM mass $m$ is $m=E-\frac{2 E^{2}}{3 \pi r^{2}}$, one is able to put $\nu(E)$ in terms of $m$, i.e., $\nu(E)=$ $\frac{2}{\sqrt{3 \pi r}} \exp \left(-\frac{\pi^{2} r^{3}}{4}+2 \pi r m\right)$. Also, since in this approximation the cavity radius and the event horizon are very close to each other, one can take $r=r_{+2}$, use $m=\frac{3 \pi}{8} r_{+}^{2}$, and find that the density of states is well approximated by

$$
\nu(E)=\frac{2}{\sqrt{3 \pi r}} \mathrm{e}^{\frac{A_{+2}}{4}},
$$

where $A_{+2}=2 \pi^{2} r_{+2}^{3}$ is the black hole's surface area. In statistical mechanics the relation between entropy $S$ and the density of states $\nu$ is $S=a \ln \nu$, for some factor $a$, so we find that the black hole entropy is

$$
S=\frac{A_{+2}}{4},
$$

as one would expect. Interestingly, this result cannot be obtained if instead of $r_{+2}$ one employs the instanton $r_{+1}$ and uses $I\left(r_{+1}\right)$ as given in Eq. $13 p$ in the partition function $Z=e^{-I\left(r_{+1}\right)}$ and then calculates the Laplace transform for $\nu(E, r)$, since the integral is divergent. Only the large black hole $r_{+2}$ makes sense in this setting.

\section{Acknowledgments}

We thank Charles Robson for conversations. RA acknowledges support from the Doctoral Programme in the Physics and Mathematics of Information (DP-PMI) and the Fundação para a Ciência e Tecnologia (FCT Portugal) through Grant No. PD/BD/135011/2017. JPSL acknowledges FCT for financial support through Project No. UIDB/00099/2020.

\section{Appendix A: Connection to York's four-dimensional results}

In extending York's four-dimensional canonical black hole thermodynamics results to five dimensions, there are several points that are worth mentioning.

When working out the possible black hole radii for a given cavity radius $r$ and a given cavity temperature $T$, one finds that in four dimensions one needs to solve a cubic polynomial with two real radii, yielding the two black holes. On the other hand, in five dimensions one instead has a quartic potential, and one would expect a different number of real solutions, but there are also only two, with radii $r_{+1}$ and $r_{+2}$, yielding again two possible black holes.

The formalism also shows that the two black holes, the small $r_{+1}$ and the large $r_{+2}$, both have a BekensteinHawking entropy in four and five dimensions, although the whole procedure is only well defined for the larger one since it is stable and the laws of thermodynamics can be applied, whereas the smaller one is unstable and cannot be treated through thermodynamics.

There are two distinct characteristic radii that appear naturally in the canonical black hole thermodynamics. One is the photon sphere radius $r_{\mathrm{ph}}$. In four dimensions, for a given temperature $T$ of the heat bath, the photon sphere separates systems that are thermodynamically stable from systems that are unstable. In five dimensions the photon sphere also plays this decisive role, namely, the black holes are in stable equilibrium with the surrounding heat bath if their cavity radius lies inside the photon sphere radius. Being a characteristic that seems to appear in any dimension, this shows that there is some intrinsic property of the photon sphere that connects it to thermodynamic stability. However, a full explanation has not been provided yet. The other characteristic radius that appears naturally in these thermodynamic systems is the Buchdahl radius $r_{\text {Buch }}$. The Buchdahl radius is the minimum radius a spherically symmetric interior 
solution with Schwarzschild exterior can have, under certain general conditions. Remarkably, we have shown that this radius also appears as the radius for which the free energy of the stable black hole passes through zero. This happens in both four and five dimensions. Since the two junctures at which $r_{\text {Buch }}$ appears are completely different, it seems that $r_{\text {Buch }}$ signals as well some intrinsic property of the spacetime geometry.

[1] J. W. York, "Black hole thermodynamics and the Euclidean Einstein action", Phys. Rev. D 33, 2092 (1986).

[2] J. B. Hartle and S. W. Hawking, "Path-integral derivation of black-hole radiance", Phys. Rev. D 13, 2188 (1976).

[3] G. W. Gibbons and S. W. Hawking, "Action integrals and partition functions in quantum gravity", Phys. Rev. D 15, 2752 (1977).

[4] S. W. Hawking, "The path-integral approach to quantum gravity", in General Relativity: An Einstein Centenary Survey, edited by S. W. Hawking and W. Israel (Cambridge University Press, Cambridge, 1979), p. 746.

[5] D. J. Gross, M. J. Perry, and L. G. Yaffe, "Instability of flat space at finite temperature", Phys. Rev. D 25, 330 (1982).

[6] B. Allen, "Euclidean Schwarzschild negative mode", Phys. Rev. D 30, 1153 (1984).

[7] E. A. Martinez and J. W. York, "Additivity of the entropies of black holes and matter in equilibrium", Phys. Rev. D 40, 2124 (1989).

[8] H. W. Braden, J. D. Brown, B. F. Whiting, and J. W. York, "Charged black hole in a grand canonical ensemble", Phys. Rev. D 42, 3376 (1990).

[9] O. B. Zaslavskii, "Canonical ensemble for arbitrary configurations of self-gravitating systems", Phys. Lett. A 152, 463 (1991).

[10] C. S. Peça and J. P. S. Lemos, "Thermodynamics of Reissner-Nordström-anti-de Sitter black holes in the grand canonical ensemble", Phys. Rev. D 59, 124007 (1999); arXiv:gr-qc/9805004
[11] J. P. Gregory and S. F. Ross, "Stability and the negative mode for a Schwarzschild black hole in a finite cavity", Phys. Rev. D 64, 124006 (2001); arXiv:hep-th/0106220.

[12] H. S. Reall "Classical and thermodynamic stability of black branes", Phys. Rev. D 64, 044005 (2001); arXiv:hep-th/0104071

[13] J. X. Lu, S. Roy, and Z. Xiao, "Phase transitions and critical behavior of black branes in canonical ensemble", J. High Energy Phys. JHEP 01 (2011) 133; arXiv:1010.2068 [hep-th].

[14] M. Bañados, C. Teitelboim, and J. Zanelli, "Black hole entropy and the dimensional continuation of the GaussBonnet theorem", Phys. Rev. Lett. 72, 957 (1994); arXiv:gr-qc/9309026

[15] F. R. Tangherlini, "Schwarzschild field in $n$ dimensions and the dimensionality of space problem", Nuovo Cimento 27, 636 (1963).

[16] H. A. Buchdahl, "General relativistic fluid spheres", Phys. Rev. 116, 1027 (1959).

[17] M. Wright, "Buchdahl type inequalities in $d$ dimensions", Classical Quantum Gravity 32, 215005 (2015); arXiv:1506.02858 [gr-qc].

[18] F. C. P. Monteiro, General and Specific Results for $d$ Dimensional Orbits in General Relativity: From a FourDimensional Spacetime to the Large d Limit (M.Sc. thesis, Instituto Superior Técnico, Lisbon, 2015).

[19] P. T. Landsberg and A. De Vos, "The Stefan-Boltzmann constant in $n$-dimensional space", J. Phys. A 22, 1073 (1989). 